\title{
Issues in the Management of Dietary Supplement Use Among Hospitalized Patients
}

\author{
Edward W. Boyer, $M D, P h D^{\text {a }}$ \\ a Department of Emergency Medicine, University of Massachusetts Memorial Medical Center, Worcester, MA, Children's Hospital, \\ Boston, MA. Email: Edward.boyer@tch.harvard.edu
}

REPRINTED FROM THE INT J MED TOXICOL 2002; 5(1): 1.

\begin{abstract}
Herbs and dietary supplements (HDS) are in widespread use in the United States, and are especially prevalent among chronically ill patients. This population is more likely to be admitted to health care facilities where they may continue to use HDS. The risk of adverse effects from HDS used during admission places significant liability on clinicians and hospitals. We describe the response of health care facilities in the Boston, Massachusetts area to this problem, and propose elements of a hospital policy which increases patient safety and satisfaction and minimizes the likelihood for adverse effects from HDS.
\end{abstract}

\section{INTRODUCTION}

Complementary and alternative medicines (CAM), whose sales reached \$US 27 billion in 1997, are in widespread use in the United States [1-4]. One subclass of CAM, herbs and dietary supplements (HDS), are especially popular. These products include herbs, minerals, teas, vitamins, animal tissue extracts, essential oils, and Asian proprietary medicines [5,6]. Patients use HDS for a variety of reasons. Many are not dissatisfied in general with conventional medicine, but instead enjoy autonomy in making health care decisions $[1,7]$. Some feel that HDS emphasize health rather than illness, or perceive these remedies to have efficacy in treating medical conditions [1,7]. Many patients believe that by being "natural", HDS are safe, and correctly observe that most herbs have no toxicity when ingested, even in overdose $[7,8]$. The public's perceptions are bolstered by aggressive promotions describing the benefits of HDS and from sophisticated marketing efforts developed by the dietary supplements industry, which vends their products alongside prescription and nonprescription pharmaceuticals $[8,9]$. This practice creates the impression of potency for HDS that equals that of pharmaceuticals [8].

As reliance upon HDS increases, clinicians and health care facilities are confronted with the issue of how to govern HDS use among patients who want to continue to use them during hospitalizations [10]. Unfortunately, the process of adapting hospital policies to accommodate these patients is complicated by federal legislation related to HDS, the lack of safety data, actions by accreditation agencies, and the inapplicability of standard pharmacy practices [10].

\section{Issues Related to Dietary Supplement Safety and Regulation}

The Dietary Supplement Health and Education Act (DSHEA), enacted by the US Congress in 1994, classifies HDS as foods regardless of historical uses [6]. Paradoxically, Congress passed this legislation after the recognition that a contaminated dietary supplement (L-tryptophan) caused the eosinophilia-myalgia epidemic $[11,12]$. This landmark legislation contains several unconventional provisions. For example, it places on the Food and Drug Administration (FDA) the burden of proving that each ingredient in a HDS product is unsafe. This is distinct from the approval process for pharmaceuticals where manufacturers must demonstrate that a product is safe and effective for its intended use before it is sold [6]. Furthermore, DHSEA prevents the FDA from requiring manufacturers to accurately identify the contents

Keywords: dietary supplements, drug interactions, inpatients 
of HDS products or the concentration of pharmacologically active agents $[6,10]$. Under a worst-case scenario, a manufacturer may claim that a product contains a specific herb, but with federal oversight of the industry restricted by DHSEA, the same product could contain a specific herb that is toxic, an herb that could produce adverse events if misused, a formulation that could produce drug-herb interactions, or contain adulterantsand be legal nonetheless $[6,10,13-17]$.

Because DSHEA severely restricts the FDA from policing truth-in-labeling, manufacturers are permitted to use vague terms to suggest therapeutic effect for nonspecific entities [8]. From vendor's claims, however, patients often extrapolate effectiveness in the management of specific medical conditions, treating virtually every medical condition with HDS [6,7]. The use of CAM is most prevalent among patients suffering from recurrent, chronic or incurable conditions such as allergies, asthma, behavioral and developmental problems, cancer, cystic fibrosis, HIV, and rheumatoid arthritis [2,18-27]. Two-thirds of chronically ill patients use HDS, frequently in conjunction with prescription and over-the-counter medications. However, only 30\% discuss their use of HDS with their physician [2,18-26,28,29].

Several studies have shown that chronically ill patients are more likely to use HDS, be admitted to a health care facility, and to continue to use HDS after admission [7,10,18,30]. Even though HDS use is more prevalent among inpatients in healthcare facilities than among the general population, its use often goes unrecognized because physicians infrequently ask patients about their use of unconventional medicines [7,10,18,30]. Those wanting to continue using HDS after admission are compelled to import their own supply of medications into a health care facility because hospital pharmacies infrequently stock HDS as formulary items [31,32]. This practice, known in pharmacy parlance as using medications from "home supply", makes the identification of HDS use among patients admitted to a health care facility particularly important [10].

\section{Policy Responses to Herbal Product and Dietary Supplement Use Among Hospital Inpatients}

Despite the absence of safety and efficacy data for most HDS, these products frequently contain pharmacologically active agents capable of producing biological responses [10]. Although not considered drugs by the Food and Drug Administration, HDS nonetheless meet the broad definition of a drug given by regulatory bodies such as the Joint Commission on Accreditation of Healthcare Organizations (JCAHO) [33]. Hospitals are therefore expected to manage HDS from home supply with the same diligence and care given to other medications brought into a health care facility by patients or their families $[10,33]$. To accreditation agencies, the use of HDS should be subjected to medical staff approval and governed by policies that regulate nonprescription medications from home supply [10].

For a patient to use a prescription or over-the-counter home supply medication drawn from home supply, hospital pharmacies typically follow established written protocols [10]. During the intake process, a physician identifies a patient's use of conventional pharmaceuticals and, if appropriate, discusses the use of home supply medications with the patient. The physician then may write an order allowing the use of medications from home supply. The nursing staff is expected to collect the patient's medicine and send it to the hospital pharmacy. The pharmacist, using a pill identification system such as IdentidexTM, must then verify the identity of the medication. Pharmacists relabel the medication and return it to the patient's floor where it is stored in the medication room or cart. The nurses administer the medication according to the dose and schedule ordered by the physician; they document the patient's use in the Medication Administration Record (MAR). Recently, healthcare organizations have struggled to apply these procedures to HDS [10].

Applying standard hospital policies to HDS is challenging. Physicians rarely identify HDS use among inpatients $[2,18-26,28,29]$. Few alternative therapies have objective data from well-designed, properly conducted trials to support their use [10]. A paucity of information compromises clinicians' ability to inform patients about the risks and benefits of HDS, so that even if physicians discover a patient's regular use of HDS, there is often insufficient evidence to support informed decision-making regarding these products [10]. As exact identification of an HDS is sought, pharmacists frequently are unable to confirm its composition or its manufacturer. Pharmacists' tasks are further complicated by drug concentrations that vary greatly between lots and manufacturers $[6,13,15]$. Finally, using the hospital nursing staff to administer potentially toxic HDS of uncertain benefit may be unethical as well as place significant liability on health care facilities and physicians [10,34].

The development of adverse effects from inpatient HDS use reported to our regional poison control center prompted us to craft a policy governing home supply use of HDS among admitted patients $[35,36]$. To determine the ways in which hospitals governed the use of HDS from home supply, we interviewed in February 2001 the directors $(\mathrm{N}=11)$ or clinical supervisors of pharmacy operations $(\mathrm{N}=3)$ for 14 Boston area hospitals, seven of which were affiliated with Harvard Medical School. We had a response rate of $100 \%$. We found significant variation in the responses generated by referral medical centers, general hospitals, community hospitals, Veteran's Administration hospitals, pediatric, ophthalmologic, and rehabilitation hospitals, and psychiatric facilities. None stocked HDS as formulary items in their hospital pharmacy. Every hospital surveyed had generated a response to inpatient use of HDS from home supply, but because of the difficulties encountered in drafting a policy, only six had placed their response in writing. Based on our survey, the ways in which hospitals manage inpatient use of HDS from home supply can be classified as follows.

One method has been to categorically ban HDS use among inpatients [10]. This strategy, however, carries the risk of patients' withholding of important information from caregivers, of alienating patients, or their smuggling of HDS into the hospital. Although partial rejection of conventional care is thought to 
be uncommon, consequences of alienation include delayed presentation during future exacerbations of illness, avoidance of conventional medical care, premature termination of care, or transfer of care to facilities that permit HDS use [10]. Unfortunately, forbidding inpatient HDS use fails not only because of the behavior of patients, but also from that of hospitals themselves. Recognizing the degree to which patient satisfaction depends upon these remedies, at least one facility has a written policy that categorically bans all HDS use, but this policy can be overridden by the written order of the senior medical staff.

A second approach has been to develop a policy that permits HDS use that is, in essence, unfettered. Facilities utilizing this approach permit HDS but did not ascribe to the accepted protocols designed to ensure the safety of home supply items. Hospitals that do not require HDS to be identified, allow patients to take any HDS irrespective of risk, or hazard inpatient overdose by not securely storing the HDS, do not meet the guidelines established by JCAHO requiring these products to be handled with the same diligence and care afforded to pharmaceutical agents.

A third approach has been to permit restricted use of HDS. One general hospital did not permit HDS. Inpatients at that facility, however, may request that their attending physician ask the Pharmaceutical and Therapeutic (P\&T) committee to add an HDS to the hospital formulary after the review of pertinent safety and efficacy data. This strategy gives the appearance of responding to patient preferences, but since the approval of a new drug by P\&T committees often requires considerable time, this approach functionally bans HDS by inpatients during a single hospitalization [10].

Hospitals interested in drafting coherent policies to control the use of HDS from home supply find that resources to assist them are lacking. Even though JCAHO clearly impels policy development, it provides no specific suggestions on the matter [10]. Additionally, to our knowledge, no Federal agenciesincluding those which foster research into complementary and alternative therapies-offer substantive assistance for policy development. The problems created by DHSEA and the absence of guidance creates striking variations in hospitals' approach to this problem, even leading to multiple contradictory policies within a single academic institution [10].

\section{Proposed Elements of a Hospital Policy Governing the Use of HDS From Home Supply}

We believe that a reasonable and enforceable policy for governing use of HDS from home supply among hospitalized patients should be based upon established pharmacy practices. It should contain the following elements: 1) a physician's order, including name and source of HDS, dose, and schedule; 2) recording, identification, and labeling of the HDS by the hospital pharmacy; 3 ) securely storing the HDS in the medication room or cart; 4) administration by patient/caretaker, with documentation by the nursing staff in the medication administration record; and 5) reporting adverse events to internal resources (hospital pharmacy and risk management personnel) and external organizations (such as Medwatch, operated by the United States Food and Drug Administration). We also believe that any policy should contain provision for educational resources to assist clinicians in making decisions and educating patients regarding HDS use.

At Children's Hospital, Boston, we have created the following protocol (Figure 1). Although drafted and revised by the primary author, it was reviewed by the directors of pharmacy and nursing services, the Pharmaceutical and Therapeutics (P\&T) committee, and attorneys for the Risk Management Foundation of Harvard University. In this policy, physicians must identify HDS use; preprinted admission history and physical forms prompt physicians to inquire about patients' regular usage. For a patient to continue receiving HDS, a physician must write an order with the dose and schedule of the HDS. Although consultation is not required, clinicians are reminded to utilize educational services such as medical toxicology, holistic medicine services, and online pharmacy databases. The HDS is sent to the pharmacy (which, since HDS cannot be identified, does not relabel it). Instead, the pharmacy maintains its own longitudinal record of patients' HDS use. The HDS is locked in the medication room until the dosing schedule calls for its administration. Nurses deliver the HDS to the patient or caretaker for administration; the nurses directly observe the use of the HDS and record this event in the medication administration record. Adverse events are reported to the hospital's risk management organization as well as Medwatch.

Each aspect of our proposed policy is designed to expand clinicians' understanding of HDS use, engage patients, or adhere to directives by credentialing organizations. For example, the educational services are intended to help physicians and patients broaden their knowledge of the clinical effects of HDS use. The use of physician's orders, pharmacy and nursing documentation, and secure storage of HDS improves compliance with accreditation requirements. Pharmacy documentation that is independent of the inpatient medical record allows facile identification of HDS use should the patient visit other clinical settings such as day surgery and outpatient clinics. Patients' sense of participating in medical care is improved by having patients/caretakers administer HDS; moreover, this practice relieves the legal and ethical burden placed on the nursing staff of having to administer HDS with unknown benefits and toxicities.

We do not ask patients to provide informed consent regarding the use of HDS. Some authorities have suggested that the absence of safety and efficacy data prevents informed consent from being applied to HDS [10]. Furthermore, asking patients to provide signed releases may offend patients, leading to surreptitious HDS use, and it may not affect liability [10].

In our facility, we have banned all members of a class of HDS known as Asian patent medications. These medications commonly contain adulterants such as heavy metals (such as mercury, arsenic and lead), pharmaceuticals (such as acetaminophen or diazepam), or materials banned for sale in this country (such as phenylbutazone and phenformin) [6]. We believe that Asian patent medications are sufficiently dangerous to warrant their exclusion from our facility. We considered banning ayurevedic 


\section{Identify herbal product/dietary supplement use}

Defined as per Dietary Supplement Health and Education Act of 1994 Pub. L. 103-417, 108 Stat. 4325, 1994.

"Use of herbal product or dietary supplement" is defined as occurring within last 48 hours. Physician must identify HDS use.

Information about use is analogous to medication use and is recorded in chart, nursing assessment tools, and progress notes.

If such a HDS is to be used, its administration must be ordered by written physician order. Toxicology service is consulted to support clinical decision making.

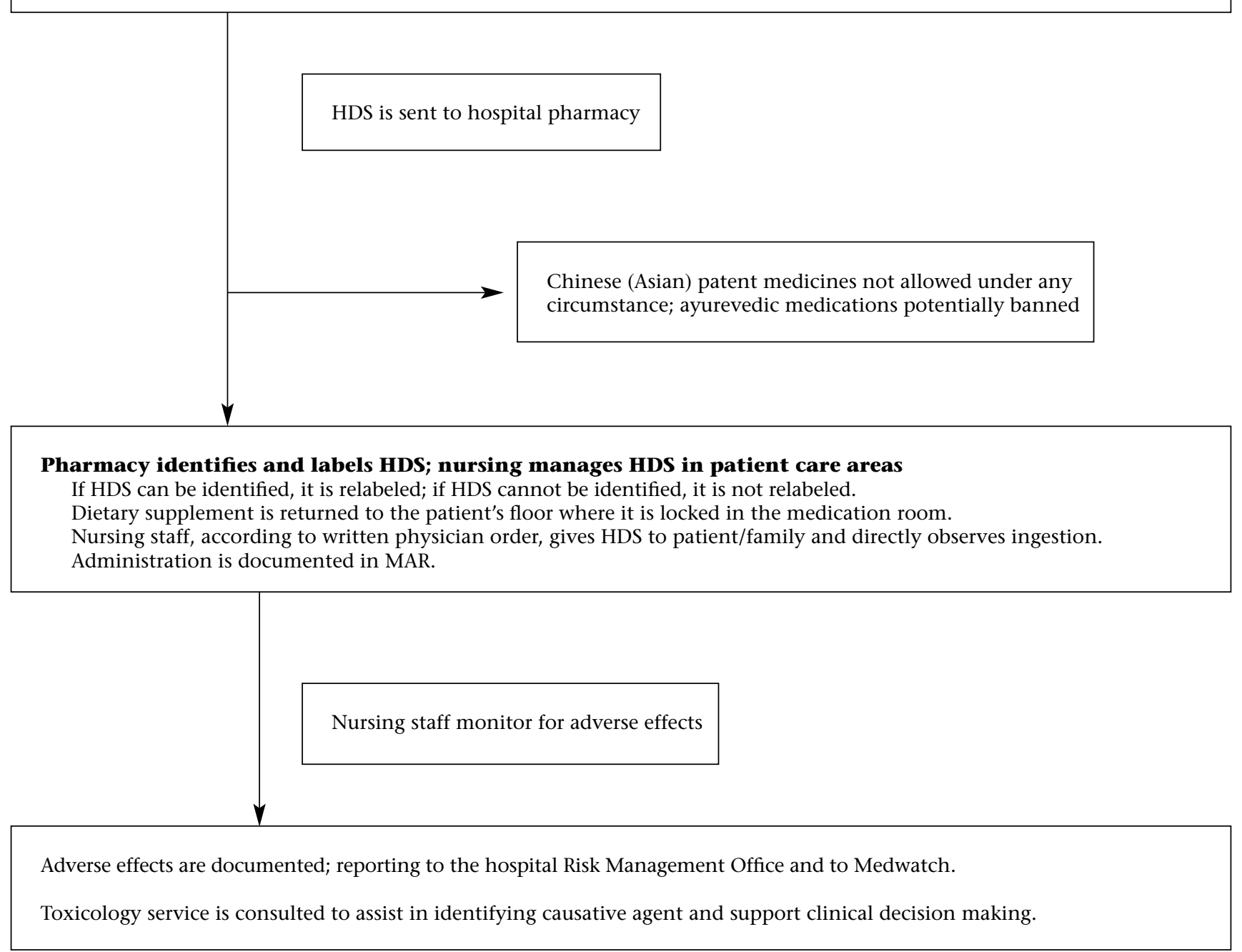

Figure 1. Children's Hospital of Boston protocol for handling home supply dietary supplements among inpatients.

medications from use in Children's Hospital, but did not because of limited evidence of broad potential for poisoning.

Because of the incongruities related to HDS, we recognize that this policy is not foolproof. We hope to stimulate the crafting of hospital policies that improve physician awareness of HDS use and that augment our understanding of the ways in which these remedies are used. Most importantly, we hope this effort increases patient safety and minimizes the potential for adverse effects from HDS.

\section{REFERENCES}

1. Eisenberg DM, Kessler RC, Foster C, Norlock FE, Calkins DR, Delbanco TL. Unconventional medicine in the United States. Prevalence, costs, and patterns of use. N Engl J Med 1993; 328:246-52.

2. Eisenberg DM, Davis RB, Ettner SL, et al. Trends in alternative medicine use in the United States, 1990-1997: results of a follow-up national survey. JAMA 1998; 280:1569-75. 
3. Bennet J, Brown C. Use of herbal remedies by patients in a health maintenance organization. J Am Pharm Assoc 2000; 40:353-358.

4. Elder N, Gillchrist A, Minz R. Use of alternative health care by family practice patients. Arch Fam Med 1997; 6:181-184.

5. Shannon M. Herbal, traditional, and alternative medicine. In: Haddad LM SM, Winchester J, ed. Clinical Management of Poisoning and Drug Overdose. Philadelphia: WB Saunders, 1998.

6. Ko R. Causes, epidemiology, and clinical evaluation of suspected herbal poisoning. Journal of toxicology-Clinical toxicology 1999; 37:697-708.

7. Astin J. Why patients use alternative medicines. JAMA 1998; 279:1548-1552.

8. Shannon M. Symposium on natural products toxicology. Journal of Toxicology-Clinical Toxicology 1999; 37:695-696.

9. Committee on Children With Disabilities. Counseling families who choose complementary and alternative medicine for their child with chronic illness and disability. Pediatrics 2001; 107:598-601.

10. Walker P. Evolution of a policy disallowing the use of alternative therapies in a health system. Am J Health-Syst Pharm 2000; 57:1984-1990.

11. Dietary Supplement Health and Education Act of 1994. Pub. L. 103-417, 108 Stat. 4325, 1994.

12. Shannon M. Alternative medicines toxicology: A review of selected agents. Journal of Toxicology-Clinical Toxicology 1999; 37:709-713.

13. Robbers J, Tyler V. The therapeutic use of phytomedicinals. In: Tyler V, ed. Tyler's herbs of choice. New York: Hawthorne Herbal, 1999.

14. Ko R. Adulterants in Asian patent medicines. N Engl J Med 1998; 339:847.

15. Hung O, Shih R, Howland M. Herbal preparations. In: Goldfrank L, ed. Goldfrank's Toxicologic Emergencies. Stamford, CT: Appleton and Lange, 1998:1221-1238.

16. Espinoza EO, Mann M-J, Bleasdell B. Arsenic and mercury in traditional Chinese herbal balls. New Engl J Med 1995; 333:803-804.

17. Lau K, Lai C, Chan A. Phenytoin poisoning after using a Chinese proprietary medicine. Hum Exp Toxicol 2000; 19:385-386.

18. Spigelblatt L, Laine-Amamara G, Pless I, Guyver A. The use of alternative medicine by children. Pediatrics 1994; 94:811-814.

19. Stern R, Canda E, Doershuk D. Use of nonmedical treatment by cystic fibrosis patients. J Adolesc Health 1992; 13:612-615.

20. Hung O, Shih R, Chiang W, Nelson L, Hoffman R,
Goldfrank L. Herbal Medication Use Among Urban Emergency Department Patients. Acad Emerg Med 1997; 4:209-213.

21. Bausell R, Lee W, Berman B. Demographic and healthrelated correlates to visits to complementary and alternative medicine providers. Med Care 2001; 39:190-196.

22. Fernandez T, Slayton W, Allen L. Use of alternative therapies for children with cancer. Pediatrics 1997; 100:1.

23. Kestin M, Miller L, Littlejohn G, Wahlqvist M. The use of unproven remedies for rheumatoid arthritis in Australia. Med J Aust 1985; 143:516-518.

24. Southwood T, Malleson P, Roberts-Thompson P, Mahy M. Unconventional remedies used for patients with juvenile arthritis. Pediatrics 1990; 85:150-4.

25. Stern R, Canda E, Doershuk C. Use of nonmedical treatment b cystic fibrosis patients. J Adolesc Health 1992; 13.

26. Fairfield K, Eisenberg D, Davis R, Libman H, Phillips R. Patterns of use, expenditures, and perceived efficacy of complementary and alternative therapies in HIV-infected patients. Arch Intern Med 1994; 158:2257-2264.

27. Fernandez C, Stutzer C, MacWilliam L, Fryer C. Alternative and complementary therapy use in pediatric oncology patients in British Columbia: Prevalence and reasons for use and nonuse. J Clin Oncol 1998; 16:1279-1286.

28. Hensrud D, Engle D, Scheitel S. Underreporting the use of dietary supplements and nonprescription medications among patients undergoing a periodic health examination. Mayo Clin Proc 1999; 74:443-447.

29. Sibinga E, Ottolini M, Duggan A, Wilson M. Communication about complementary/alternative medicine use in children. Pediatr Res 2000; 47:226A.

30. Fraenkel M, Arye E. The growing need to teach about complementary and alternative medicines: questions and challenges. Acad Med 2001; 76:251-254.

31. McCann G. Alternative medicines starting to penetrate hospital DURs. Hosp Pharm Rep 1997; 11:48-90.

32. Beal F. Herbals and homeopathic remedies as formulary items? Am J Health-Syst Pharm 1998; 55:1266-1267.

33. Rich D. Point-of-care automated dispensing devices: herbal products, proper medication orders; expiration dating. Hosp Pharm 1999; 34:989-995.

34. Boozang K. Western medicine opens the door to alternative medicine. Am J Law Med 1998; 24:185-212.

35. Boyer EW. 2000 Poisoning Data: Massachusetts Poison Control Center, 2000.

36. Boyer EW, Kearney S, Woolf A, Shannon M, Kemper K. Poisoning from a dietary supplement administered during hospitalization. Pediatrics 2002 109: e49. 